\title{
NEW AROMATIC POLYAMIDES AND POLYIMIDES HAVING AN ADAMANTANE BULKY GROUP
}

(empty line 6 pt)

Diego Plaza-Lozano ${ }^{1,2}$, Bibiana Comesaña-Gándara ${ }^{1,3,4}$, Mónica de la Viuda ${ }^{1}$, Jong Geun Seong ${ }^{4}$, Laura Palacio ${ }^{1}$, Pedro Prádanos ${ }^{1}$, José G de la Campa ${ }^{3}$, Purificación Cuadrado ${ }^{1,2}$, Young Moo Lee ${ }^{4}$, Antonio Hernández ${ }^{1, *}$, Cristina Alvarez ${ }^{3}$, Angel E. Lozano $^{1,3, *}$

(empty line 6 pt)

${ }^{1}$ SMAP UA-UVA_CSIC, University of Valladolid, Valladolid, Spain

${ }^{2}$ Department of Organic Chemistry, University of Valladolid, Valladolid, Spain

${ }^{3}$ Institute of Polymer Science and Technology, ICTP-CSIC, Madrid, Spain, ${ }^{4}$ Department of Energy Engineering, Hanyang University, Seoul 133-791, Republic of Korea

\section{ABSTRACT}

This paper reports the synthesis and characterization of a new rigid diamine monomer, having a spiro carbon moiety and an adamantane bulky group in its structure; namely spiro-(adamantane-2,9'(2',7'-diamino)-fluorene) (SADAF). After its synthesis, using a straightforward methodology, a novel family of aromatic polyimides (PIs) and polyamides (PAs) has been attained by reaction of SADAF with three aromatic dianhydrides and two diacid chlorides, respectively. Two of the polyimides were obtained through the formation of the corresponding polyamic acid and subsequent thermal cycloimidization, whereas the other one, the polyimide from SADAF and 2,2bis(3,4-dicarboxyphenyl)hexafluoropropane dianhydride (6FDA), could be synthesized by chemical imidization from the polyamic precursor. Regarding polyamides, two new ones, made from SADAF and isophthaloyl chloride (IPC) and the diacid chloride of 2,2bis(4-carboxyphenyl)hexafluoropropane (6FC) could be obtained. All the polymers showed high $\mathrm{Tg}$, above $350^{\circ} \mathrm{C}$, and excellent thermal resistance, with onset degradation temperatures higher than $450{ }^{\circ} \mathrm{C}$. Polymers formed by the combination of SADAF with 6FDA and 6FC were prepared as dense films with good mechanical properties and their permselectivity properties were measured.

Corresponding authors: tonhg@termo.uva.es and lozano@ictp.csic.es

Keywords: gas separation; adamantane; aromatic polyamides; aromatic polyimides 


\section{Introduction}

Among the wide variety of polymers employed for advanced applications, aromatic polyimides (PIs) and aromatic polyamides (PAs) are considered as polymers with high technological interest due to their good mechanical properties, excellent thermal stability, elevated chemical resistance and high glass transition temperature[14]. Furthermore, it is feasible to access to a wide variety of polyimide or polyamide structures by synthesis of new monomers or by simple modification of the polymer. By applying these modifications, polymers with tailor-made properties can be employed in high performance applications. Following this approach, it is affordable to design polymers having high fractional free volume (FFV) that show excellent properties to selectively separate mixtures of gases[5-7].This approach has been exhaustively employed for PIs even though the use of well-designed aromatic polyamides should be favored because the synthesis of PAs is less cumbersome and also their fabrication and processing cost are by far much lower than those of PIs materials[8,9]. Thus, and regarding their gas permeation properties, aromatic PAs and PIs have been improved by modification of their chemical structure, for example, introducing bulky side substituents that hinder the molecular packing[10-13]. Also, it is possible to increase, by playing with the molecular structure, the rigidity of the macromolecular backbone, which translates to an improvement of gas selectivity for a mixture of gases[14]. This combination of a loose structure and high rigidity produces materials having high permeability and good permselectivity[15,16].

The adamantane (tricyclo[3.3.1.1]decane) is a highly symmetric molecule composed of three rigid cyclohexane rings in chair-type conformation[17]. This conformation, relatively free of conformational stress, results in a large and bulky structure which tends to inhibit the packing of polymer chains and to decrease the polymer crystallinity, what is translated into an improved processability[18,19]. In addition, adamantane units have higher thermal resistance than other aliphatic moieties[19]. This moiety has been widely used as bulky group in the main backbone of polymer chains as: polysulfones, polyesters, polyamides and polyimides[20,21] and also as lateral substituent in copolyimides, poly(ether imide)s, polyacrylates, poly(phenylene)s, poly(ether ketone)s and poly(benzyl ether)s[21,22]. Therefore, it is expected that the use of new nucleophilic monomers, as diamines, easily synthesized from 2-adamantanone using electrophilic aromatic substitution reactions (SEAr) and 
ulterior modification, could be a reasonable way to improve the properties of PIs and PAs. In particular, the introduction of a bulky group conveniently placed in an aromatic diamine has permitted to increase in a synergistic way the rigidity of the molecular chain and the FFV of polyimides[23]. Also, polymer chains based on spiro structures have been studied as an interesting route to improve the thermal resistance and gas separation properties of polymers[24,25].

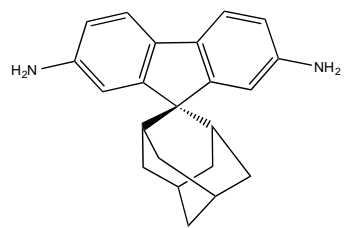

Figure 1. Spiro-(adamantane-2,9'(2',7'-diamino)-fluorene) (SADAF)

The approach in the present work is to incorporate the spiro-(2,2'adamantane-9,9'-fluorene) group in the backbone of aromatic PIs and PAs by using a very rigid and voluminous diamine. Formally, the designed diamine is related to a 4,4'diaminobiphenyl structure but with a severe rotational hindrance of the phenyl groups because their 2 and $2^{\prime}$ positions are blocked by attaching them, through a spiro $\mathrm{sp}^{3}$ carbon, to the 2 position of a bulky adamantane group, as it can be seen in Fig. 1. The reactivity of the diamine spiro-(adamantane-2,9'(2',7'-diamino)-fluorene) (SADAF) was estimated calculating its Highest Occupied Molecular Orbital (HOMO) energy, where higher energy means higher reactivity, by the semiempirical method AM1[26]. The HOMO energy of SADAF (-7.73 eV) was higher than that of benzidine $(-7.94 \mathrm{eV})$, and lower than that of $p$-phenylene diamine $(-7.32 \mathrm{eV})$. Benzidine and $p$-phenylene are very reactive diamine monomers that produce high molecular weight PIs and PAs that are rather insoluble, unless hinged, nonplanar dianhydride or diacid chloride are used[27]. Thus, it was expected that the reactivity of diamine SADAF would be enough to obtain high molecular weight polymers, in which the segmental mobility restriction by spiro $\mathrm{sp}^{3}$-carbon would impart a significant increase in glass transition temperature, $\mathrm{Tg}$, and thermal stability. Moreover, the presence of the bulky adamantane group should cause an effective chain separation affording materials with high FFV and good solubility in organic solvents[20]. It has been recently reported that analogous polyimides containing 
spirobifluorene in the main chain showed high solubility, high thermal stability and good mechanical properties[28,29].

In this work, we report the synthesis of diamine SADAF using a straight-forward synthetic methodology, which has produced a high-purity monomer in reasonable yield, and the preparation of new PIs and PAs from this diamine and aromatic dianhydrides, such as; pyromellitic dianhydride (PMDA), biphenyl tetracarboxylic dianhydride (BPDA) and 2,2-bis(3,4-dicarboxyphenyl)hexafluoropropane dianhydride (6FDA), or by reaction with diacid chlorides, such as isophthaloyl chloride (IPC) and 2,2-bis(4carboxyphenyl) hexafluoropropane diacid chloride (6FC). Based on our previous experience, the synthesis of polymers was carried out by in situ silylation of the diamine that is a very efficient activation method to yield high molecular weight polymers even for diamines with low reactivity or with steric hindrance[30-33]. Unfortunately, attempts to produce the very rigid (rod-like) polyamide from SADAF and terephthaloyl chloride (TC) were unsuccessful.

It was expected that the combination of high backbone rigidity and high FFV in the resulting polymers would lead to better gas separation properties. Particular attention was paid to characterize the polymers by spectroscopic methods and to evaluate thermal and mechanical properties in order to figure out the real capabilities of the synthesized polymers as gas separation membranes. Only those polymers able to form dense membranes from solutions and having good mechanical properties were tested with pure gases $\left(\mathrm{He}, \mathrm{O}_{2}, \mathrm{~N}_{2}, \mathrm{CH}_{4}, \mathrm{CO}_{2}\right)$ and their gas separation performances were compared with other polymers with related structure[21,34-36].

\section{Experimental}

\subsection{Materials}

Anhydrous 1-methyl-2-pyrrolidinone (99.5\%, NMP) and anhydrous N,Ndimethylacetamide $(99.5 \%$, DMAc) were used as received from Sigma-Aldrich (Milwaukee, WI, USA).

4,4'-(hexafluoroisopropylidene)diphthalic dianhydride (99\%, 6FDA) was purchased from ABCR (GmbH \& Co. KG, Karlsruhe, Germany) and it was sublimed at 
$220{ }^{\circ} \mathrm{C}$ under high vacuum just before its use. Pyromellitic anhydride (PMDA) and biphenyl tetracarboxylic dianhydride (BPDA) were obtained from Aldrich and they were purified by sublimation at high temperatures $\left(210{ }^{\circ} \mathrm{C}\right.$ and $240{ }^{\circ} \mathrm{C}$, respectively) under vacuum just prior their use. Isophthalic acid dichloride (IPC) was purchased from Aldrich and purified by recrystallization in hexane and finally by sublimation at $50{ }^{\circ} \mathrm{C}$ under vacuum. High-purity 2,2-Bis(4-carboxyphenyl) hexafluoropropane (6FC) was obtained from Chriskev ( Overland Park, KS, USA).

\subsection{Monomer synthesis}

Synthesis of high purity 2,2-bis(4-carboxyphenyl)hexafluoropropane diacid chloride (6FC) was carried out by using a methodology published elsewhere[37]

Spiro-(adamantane-2,9'(2',7'-diamino)fluorene) (SADAF) (4) was obtained using the synthetic methodology depicted in Scheme 1. Detailed synthesis of SADAF is described in the Supporting Information part.

\subsection{Synthesis of polyimides (PIs) and membrane preparation}

A series of polyimides was synthesized from the new diamine SADAF (4) and three commercial dianhydrides, PMDA, BPDA and 6FDA, via an activated polycondensation method. In a first stage, the poly(amic acid) was obtained by reacting the dianhydride with the diamine through a base-assisted in situ silylation of the diamine. In a second stage, the cyclodehydration to polyimide was carried by using a chemical imidization protocol for polyimide 6FDA-SADAF, and by using a cyclodehydration thermal process for the polyimides PMDA-SADAF and BPDASADAF. Yields were almost quantitative for all polyimides.

The following route was used for the preparation of poly(amic acid)s: a threenecked flask of $50 \mathrm{~mL}$, equipped with a mechanical stirrer and nitrogen inlet and outlet, was charged with SADAF (4) (5.0 mmol, $1.5850 \mathrm{~g})$, and $5.0 \mathrm{~mL}$ of anhydrous NMP. The mixture was stirred at room temperature under a blanket of nitrogen until the solid was entirely dissolved. The solution was then cooled to $0{ }^{\circ} \mathrm{C}$ and $11.0 \mathrm{mmol}$ of anhydrous pyridine $(0.880 \mathrm{~g})$ and $11.0 \mathrm{mmol}$ of CTMS $(1.198 \mathrm{~g})$ were slowly added. 
The solution was allowed to warn up to room temperature and stirred for 15 min to assure the formation of the silylated diamine. It was once more cooled to $0{ }^{\circ} \mathrm{C}$ and 5.0 mmol of dianhydride was rapidly added followed by $5.0 \mathrm{~mL}$ of anhydrous NMP and $1.10 \mathrm{mmol}$ of DMAP as co-base $(0.1344 \mathrm{~g}, 10 \%$ of DMAP relative to moles of Py). The reaction mixture was stirred for $15 \mathrm{~min}$ at $0{ }^{\circ} \mathrm{C}$ and then the temperature was slowly raised up to room temperature and left overnight to form the poly(amic acid) solution.

\subsubsection{Chemical imidization (CI) of polyimides}

The chemical imidization of poly(amic acid) derived from dianhydride 6FDA was accomplished by adding $40 \mathrm{mmol}$ of acetic anhydride, $(4.116 \mathrm{~g})$ and $40 \mathrm{mmol}$ $(3.164 \mathrm{~g})$ of anhydrous pyridine to the previous poly(amic acid) $(5 \mathrm{mmol})$ solution and stirring at room temperature for $6 \mathrm{~h}$, followed by heating for one additional hour at 60 ${ }^{\circ} \mathrm{C}$. When cooled to room temperature, the polymer solution was dropwise poured into

$1 \mathrm{~L}$ of deionized water, washed several times with deionized water and ethanol to remove traces of solvent and oligomers. The polyimide was then dried overnight at 120 ${ }^{\circ} \mathrm{C}$ under vacuum.

Dense membrane was prepared by casting from $8 \%(\mathrm{w} / \mathrm{v})$ solution of soluble polyimide in DMAc (filtered through a $3.1 \mathrm{~mm}$ PTFE fiberglass), onto a glass plate, at $60{ }^{\circ} \mathrm{C}$ overnight. The film was stripped off from the glass plate and dried in an oven under vacuum at $120{ }^{\circ} \mathrm{C}$ for $12 \mathrm{~h}$. Afterwards, the polyimide film was slowly heated at $350{ }^{\circ} \mathrm{C}$, maintained to this temperature for $5 \mathrm{~min}$ in a vacuum oven, and allowed to cool slowly into the oven. Film thickness was between 50-55 $\mu \mathrm{m}$.

\subsubsection{Thermal imidization (TI) of polyimides}

Dense membranes of PMDA-SADAF and BPDA-SADAF were prepared by casting process from their poly(amic acid) solutions. The solutions were diluted with 10 $\mathrm{mL}$ NMP ( $1 \mathrm{~mL}$ of NMP per mL of polyamic solution), filtered through a $3.1 \mu \mathrm{m}$ PTFE fiberglass, spread on a glass plate and left at $80{ }^{\circ} \mathrm{C}$ overnight to remove most of the solvent. The poly(amic acid) films were then imidized by the following sequential heating under nitrogen atmosphere: $120^{\circ} \mathrm{C} / 12 \mathrm{~h}, 150{ }^{\circ} \mathrm{C} / 1 \mathrm{~h}, 200{ }^{\circ} \mathrm{C} / 1 \mathrm{~h}, 250{ }^{\circ} \mathrm{C} / 1 \mathrm{~h}$, $300{ }^{\circ} \mathrm{C} / 30 \mathrm{~min}$ and $350{ }^{\circ} \mathrm{C} / 10 \mathrm{~min}$. They were allowed to cool slowly into the oven. The complete imidization was confirmed by ATR-FTIR. Film thickness was between 40-50 $\mu \mathrm{m}$. 


\subsection{Synthesis of polyamides (PAs) and membrane preparation}

The synthesis of aromatic polyamides, denoted as IP-SADAF and 6F-SADAF, was carried out by reacting the in situ silylated diamine and diacid chlorides, IPC and 6FC in a polar aprotic solvent[30]. The synthesis of the rod-like polyamide made from terephthaloylchloride and SADAF was attempted. However, premature precipitation of the polymer was observed (even including in the solution $5 \% \mathrm{w} / \mathrm{v}$ of $\mathrm{LiCl}$ ).

The synthetic route to obtain polyamides from SADAF (4) was as follows: A three-necked flask, equipped with a mechanical stirrer and nitrogen inlet and outlet, was charged with diamine $4(3.1700 \mathrm{~g}, 10.0 \mathrm{mmol})$ and $10 \mathrm{~mL}$ of anhydrous DMAc. The diamine was allowed to dissolve for approximately $15 \mathrm{~min}$ before making its silylation by adding $22 \mathrm{mmol}(1.760 \mathrm{~g})$ of anhydrous Py and $22 \mathrm{mmol}$ of CTMS (2.396 g). After $30 \mathrm{~min}, 10.0 \mathrm{mmol}$ of the corresponding diacid chloride was added followed by 3.3 mmol $(0.4032 \mathrm{~g})$ of DMAP as co-base (15\% of DMAP relative to moles of Py) and 10 $\mathrm{mL}$ of anhydrous DMAc. The components were allowed to react for $24 \mathrm{~h}$ at room temperature. The highly viscous solution was poured dropwise into $1 \mathrm{~L}$ of deionized water. The resulting polymer was filtered off, washed repeatedly in hot water and subsequently in an ethanol-water mixture (1/1 ratio). Finally, polymer was dried at 150 ${ }^{\circ} \mathrm{C}$ under vacuum overnight.

Dense membranes of polyamides were prepared by casting. A $10 \%$ solution of polyamide in DMAc (w/v) was filtered through a $3.1 \mu \mathrm{m}$ PTFE fiberglass, spread on a glass plate, and left at $60^{\circ} \mathrm{C}$ overnight to remove most of the solvent. Polymer film was peeled off from the glass and placed into a vacuum oven at $120{ }^{\circ} \mathrm{C} / 2 \mathrm{~h}, 150{ }^{\circ} \mathrm{C} / 2 \mathrm{~h}, 180$ ${ }^{\circ} \mathrm{C} / 1 \mathrm{~h} ; 200^{\circ} \mathrm{C} / 1 \mathrm{~h}$ and $250{ }^{\circ} \mathrm{C} / 30 \mathrm{~min}$. They were allowed to cool slowly into the oven. The absence of residual solvent was confirmed by thermal gravimetric analysis (TGA).

\subsection{Measurements}

${ }^{1} \mathrm{H}$ NMR and ${ }^{13} \mathrm{C}$ NMR measurements of precursors, monomers and polymers were performed in deuterated dimethyl sulfoxide (dmso-d6, Sigma-Aldrich, Milwaukee, WI, USA) and chloroform ( $\mathrm{CDCl}_{3}$, Sigma-Aldrich, Milwaukee, WI, USA)) to confirm the structure of the materials and their purity using a Varian Innova 400 (Larmor frequencies of 400 and $100 \mathrm{MHz}$ for ${ }^{1} \mathrm{H}$ and ${ }^{13} \mathrm{C}$, respectively) (Varian, Palo Alto, CA, USA). Tetramethylsilane was used as an internal standard 
Fourier Transform Infrared Spectra (FTIR) of polymer films was recorded on a Perkin-Elmer RX-1 spectrometer with Universal ATR Sampling Accessory Module (Perkin Elmer, Boston, MA, USA).

Differential scanning calorimetric (DSC) data were obtained on a TA Instruments DSC Q-2000 Analyzer (TA Instruments, DE, USA). The experiments were conducted at a heating rate of $20{ }^{\circ} \mathrm{C} / \mathrm{min}$ under nitrogen $(50 \mathrm{~mL} / \mathrm{min})$, using approximately $6 \mathrm{mg}$ of sample in hermetic aluminum pans.

Thermogravimetric analyses (TGA) were performed on a TA Instruments Q-500 thermobalance (TA Instruments, DE, USA). Dynamic ramp scans were run at $10^{\circ} \mathrm{C} / \mathrm{min}$ under a controlled flux of nitrogen $(60 \mathrm{~mL} / \mathrm{min})$, using approximately $5 \mathrm{mg}$ of sample.

Absolute molecular weight measurements were performed on a size exclusion chromatography (SEC) system coupled with a differential refractometer detector (VE 3580 RI detector, Viscotek) and a device containing both differential viscometer (DV) and right angle light scattering (RALS) detectors (Trisec Model 270, Dual Detector, Viscotek, Houston, TX, USA). The measurements were performed on three $(30 \mathrm{~cm} \mathrm{x}$ $7.5 \mathrm{~mm}$ ID) columns packed with $500 \AA, 10^{3} \AA$ and $10^{4} \AA$ PLGEL ${ }^{\mathrm{TM}}, 5 \mu \mathrm{m}$ particle diameters (Polymer Labs). The injector and column compartments were maintained at a constant temperature of $70{ }^{\circ} \mathrm{C}$. Each sample was injected at a volume of $100 \mu \mathrm{L}$ and run in $\mathrm{N}, \mathrm{N}$-dimethylformamide (Multisolvent HPLC grade ACS ISO UV-VIS, Scharlau, Barcelona, Spain) with $0.1 \%$ lithium bromide anhydrous (Alfa Aesar) at a flow rate of $1 \mathrm{~mL} / \mathrm{min}$ using an isocratic pump system. The sample was prepared at a concentration of $5 \mathrm{mg} / \mathrm{mL}$, and it was filtered through a $0.20 \mu \mathrm{m}$ disposable Teflon filter (Symta PTFE $0.20 \mu \mathrm{m}, 17 \mathrm{~mm}$ ) before injection. The instrument constants were determined using a narrow range polystyrene standard (Polymer Labs, $M p=66000$ Dalton, $\mathrm{Mw} / \mathrm{Mn}=1.03$ ). The molecular weights and intrinsic viscosity were determined by using OmniSEC software, version 4.7 from Viscotek.

Wide-angle X-ray scattering (WAXS) of polymer films were recorded in the reflection mode at room temperature, using a Bruker D8 Advance diffractometer provided with a Goebel Mirror and a PSDVantec detector (Bruker AXS, Madison, WI, USA). $\mathrm{CuK}_{\alpha}$ radiation (wavelength of $1.54 \AA$ ) was used. A step-scanning mode was used for the detector, with a $2 \Theta$ step of $0.024^{\circ}$ and 0.5 s per step.

Film density $(\rho)$ was determined from Archimedes's principle using a toploading electronic XS105 Dual Range Mettler Toledo balance and a density kit Mettler, 
Toledo, OH, USA). The samples were sequentially weighted in air and into high purity isooctane. Six density measurements were carried out on each sample and the density was calculated as follows:

$$
\rho=\rho_{\text {liquid }}\left[\frac{\omega_{\text {air }}-\omega_{\text {liquid }}}{\omega_{\text {air }}}\right]
$$

being $\rho_{\text {liquid }}$ the density of isooctane, $\omega_{\text {air }}$ the weight of the sample in air, and $\omega_{\text {liquid }}$ the weight of the sample when is submerged in isooctane.

The density data values were used to evaluate chain packing by calculating the fractional free volume (FFV), which was estimated using the following relation:

$$
F F V=\frac{V_{e}-1.3 V_{W}}{V_{e}}
$$

where $\mathrm{Ve}$ is the polymer specific volume and $\mathrm{V}_{\mathrm{W}}$ is the van der Waals volume, which was obtained by molecular modeling of structures using the semi-empirical method Austin Model1 (AM1)[26], calculated on using the Hyperchem computer program, version8.0[38].

Mechanical properties (uniaxial tension tests) were determined on a MTS Synergie 200 apparatus fitted with a $100 \mathrm{~N}$ load cell at room temperature (MTS System Corporation, Ivry sur Seine, France). The film samples of $5 \mathrm{~mm}$ width and $3 \mathrm{~cm}$ length were clamped at both ends with an initial gauge length of $10 \mathrm{~mm}$, and the elongation rate was of $5 \mathrm{~mm} / \mathrm{min}^{-1}$. At least eight samples were tested for each film.

\subsection{Permeability measurements}

Permeability of each sample was determined using a constant volume, variable pressure permeation system. The sample permeation was tested for multiple gases, all with purity higher than $99 \%$, on a single film at 3 bar. To circumvent the influence of plasticization, $\mathrm{He}, \mathrm{O}_{2}, \mathrm{~N}_{2}$, and $\mathrm{CH}_{4}$ were tested before $\mathrm{CO}_{2}$. The sample was kept under vacuum for approximately $12-24 \mathrm{~h}$ before testing to remove any atmospheric gases previously dissolved in the film. The absence of pinholes was checked by helium permeation at three upstream pressures. 
Under conditions of steady state of permeation, the permeability coefficient, $\mathrm{P}$, was determined from the slope of the downstream pressure versus time, $d p(t) / d t$, according to the following equation:

$\mathrm{P}=\frac{273}{76} \frac{\mathrm{v} l}{\mathrm{ATp}_{O}} \frac{d \mathrm{p}(\mathrm{t})}{d \mathrm{t}}$

where $A$ and $l$ are respectively the effective area and the thickness of the film, $T$ is the temperature in $\mathrm{K}$, and $p_{o}$ is the pressure of the feed gas in the upstream chamber. $P$ is usually expressed in Barrer $\left[1\right.$ Barrer $=10^{-10}\left(\mathrm{~cm}^{3}(\mathrm{STP}) \cdot \mathrm{cm}\right) \cdot\left(\mathrm{cm}^{-2} \cdot \mathrm{s}^{-1} \cdot \mathrm{cmHg}^{-1}\right)=$ $7.5005 \cdot 10^{-18} \mathrm{~m}^{2} \mathrm{~s}^{2} \mathrm{~Pa}^{-1}$ (SI units)]. The ideal selectivity for a gas pair was calculated from the ratio of their pure gas permeabilities.

The diffusion coefficient, $D$, was obtained from the intercept with the time axis using the relation:

$$
\mathrm{D}=\frac{l^{2}}{6 \theta}
$$

where $\theta$ is the time lag.

The apparent solubility coefficient, S, was calculated from the following relation:

$$
\mathrm{S}=\frac{P}{\mathrm{D}}
$$

\section{Results and discussion}

\subsection{Synthesis of SADAF monomer (4)}

Scheme 1 outlines the synthetic route applied for the preparation of the new spiro-(adamantane-2,9'(2',7'-diamino-fluorene) monomer, SADAF (4). The synthetic protocol included 3 steps. In a first step 2-(2-biphenyl)-adamantanol (1) was efficiently synthesized from reaction of 2-adamantanone with 2-biphenylmagnesium bromide in dry ether at reflux temperature overnight under nitrogen atmosphere in $81 \%$ yield after purification by recrystallization. The compound (1) was then cyclized by refluxing with glacial acetic in the presence of acid catalyst (hydrogen chloride) in 94\% yield giving spiro-(adamantane-2,9'-fluorene) (2).

After cyclization, the nitration of compound (2) was carried out by reacting with a mixture of concentrate nitric acid and glacial acetic in $70 \%$ yield after purification. 
Finally the nitro groups of (3) were catalytically reduced to amine groups with hydrazine as the reducing agent and palladium as the catalyst, in $89 \%$ yield after purification. It could be seen that the limiting step of this synthesis relied on the nitration of the spiro-(adamantane-2,9'(2',7'-dinitro-fluorene) (3).

The obtained monomer showed, as determined by using spectroscopic methods, a high purity, which should permit to obtain high molecular weight polymers.

It should be noted that the employed synthetic methodology is effective and flexible enough to be used for the synthesis of other monomers. Use of this methodology to obtain other nucleophilic moieties will be described elsewhere.
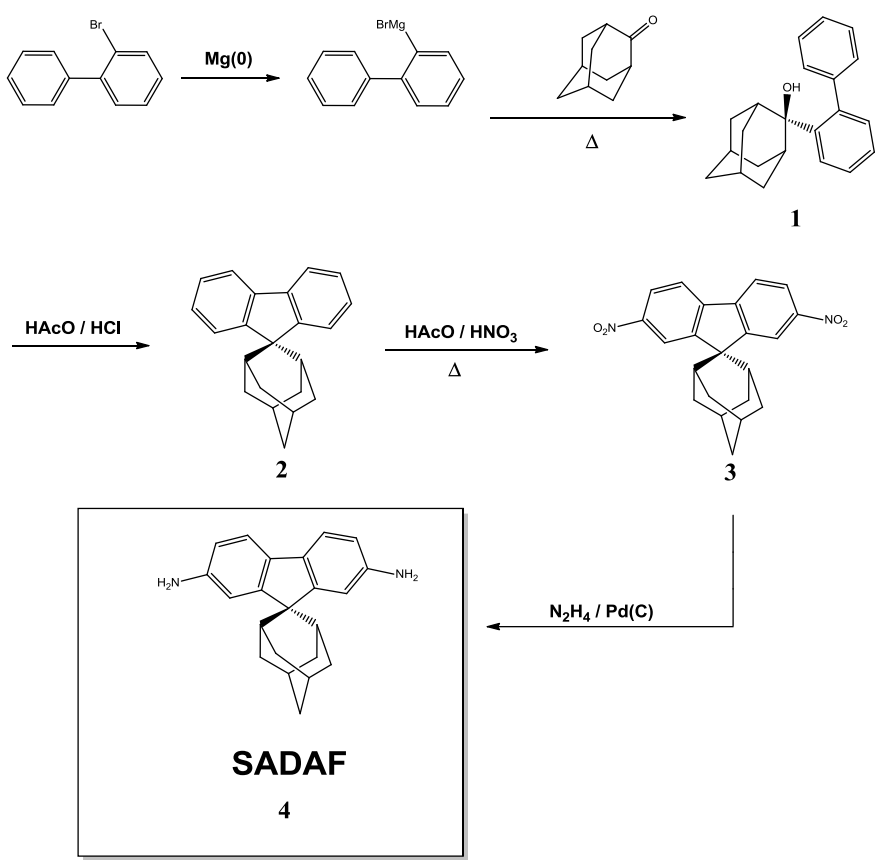

$\mathrm{N}_{2} \mathrm{H}_{4} / \mathrm{Pd}(\mathrm{C})$

Scheme 1.- Scheme of synthesis of spiro-(adamantane-2,9'(2',7'-diamino-fluorene) (SADAF) (4)

The structure of intermediates and SADAF diamine was confirmed by spectroscopic methods ( ${ }^{1} \mathrm{H}-\mathrm{NMR},{ }^{13} \mathrm{C}-\mathrm{NMR}$ and FTIR). As an example the ${ }^{1} \mathrm{H}-\mathrm{NMR}$ and ${ }^{13} \mathrm{C}-\mathrm{NMR}$ spectra of SADAF is showed in Fig. 2 included in the supporting information section. 
All the NMR peaks could be easily assigned by employing 2D NMR techniques and also by comparing these spectra with other related structures. The analysis of the resonance value of the $\mathrm{NH}_{2}$ and aromatic resonance peaks, along with the HOMO energy value, determined by using the AM1 semiempirical quantum mechanical method, denoted that the reactivity of this monomer was high enough to produce high molecular weight polymers.

3.2. Synthesis and spectroscopic characterization of polyimides (PIs) and polyamides $(P A s)$.

Aromatic polyimides (PIs) and polyamides (PAs) were synthesized by reaction of the diamine SADAF with diverse dianhydrides and diacid chlorides, respectively, whose structures are given in Schemes 2 and 3.

Aromatic polyimides were obtained through the synthesis of polyamic acids using a base-assisted reaction where the diamines were modified by using the in situ silylation method as described previously[32]. This assisted method is much more efficient in order to obtain higher molecular weight aromatic polyimides than using other polycondensation methods. The polyimide derived from 6FDA was prepared by chemical imidization (CI) of the corresponding polyamic acid precursor but the other two polyimides (PMDA-SADAF and BPDA-SADAF) only could be obtained by thermal imidization (TI) of their polyamic acids because they prematurely precipitated during the chemical conversion to polyimides. Aromatic polyamides were prepared through a classical polycondensation reaction using also the in situ silylation method as described elsewhere[30]. It should be remarked that this policondensación reaction gives aromatic polyamides having molecular weight by far much higher than those obtained from the classical reaction between aromatic diamines and aromatic diacid chlorides[39]. 


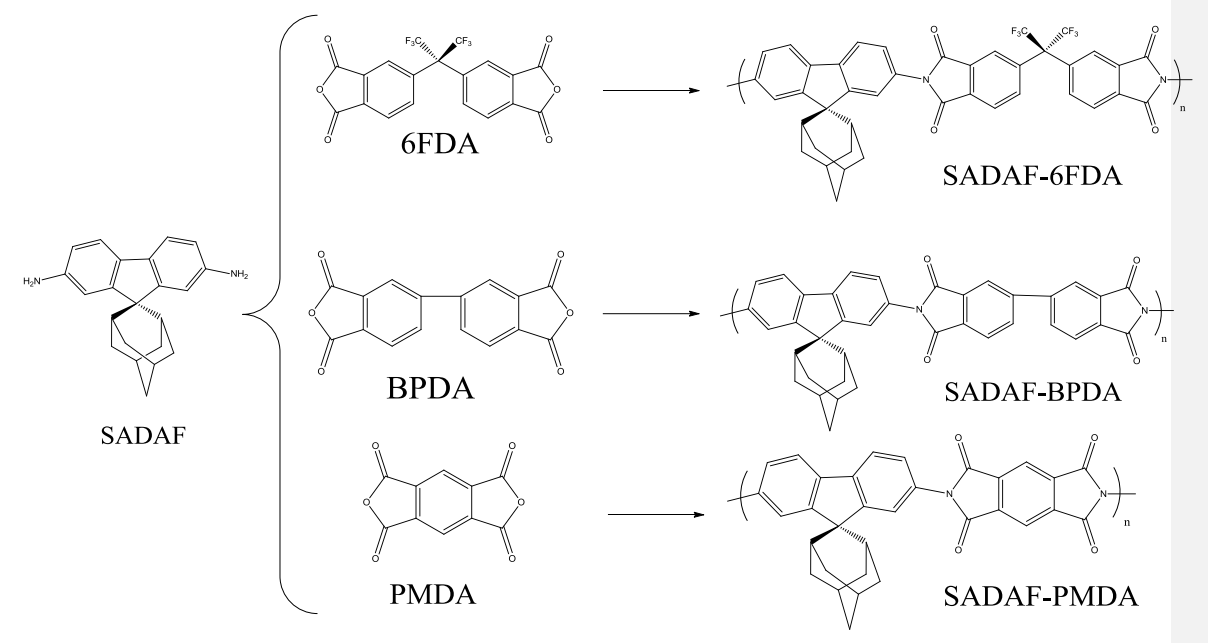

Scheme 2. Aromatic polyimides derived from SADAF. SADAF-6FDA was obtained by chemical imidization (CI). SADAF-BPDA and SADAF-PMDA were obtained by thermal imidization (TI).

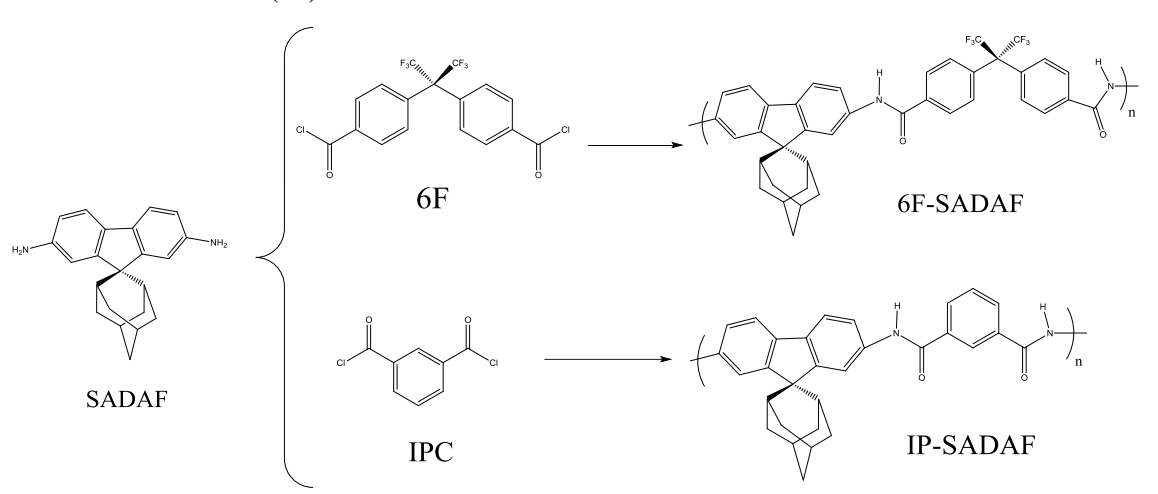

Scheme 3. Aromatic polyamides derived from SADAF

The structure of soluble polymers was checked by NMR. As an example the ${ }^{1} \mathrm{H}$ NMR spectrum for 6FDA-SADAF is included in the supporting information of this paper.

It was possible to assign the NMR peaks by comparison with the NMR spectrum obtained for the precursor monomer, and also by comparing with polymers derived from the corresponding dianhydride. 
The structure of polyimides and polyamides was also confirmed by ATR-FTIR. As an example, Fig. 2 shows the FTIR spectra of the polyimide 6FDA-SADAF and the polyamide 6F-SADAF. The presence of characteristic imide group absorptions in the regions 1778 and 1720 ( $\mathrm{C}=\mathrm{O}$ asymmetric and symmetric stretching, respectively), $1380-1350 \mathrm{~cm}^{-1}$ (C-N stretching) and 750-720 $\mathrm{cm}^{-1}$ (imide ring deformation), and the absence of the $\mathrm{C}=\mathrm{O}$ band of poly(amic acid) around $1680 \mathrm{~cm}^{-1}$ indicated that polyimides were well imidized. As to polyamides, the spectra showed the characteristic absorption of amide groups at around 3302-3334 $\mathrm{cm}^{-1}$ and 1651-1658 $\mathrm{cm}^{-1}$, NH stretching and $\mathrm{C}=\mathrm{O}$ stretching, respectively. Only the bands corresponding to the $\mathrm{C}-\mathrm{H}$ stretching around $2900 \mathrm{~cm}^{-1}$ of the adamantane moiety were clearly observed in both polymers. The rest of the characteristic vibrational bands of adamantane, such as $\mathrm{CH}_{2}$ scissoring around $1450 \mathrm{~cm}^{-1}$, and $\mathrm{CH}_{2}$ rocks, wags and twists in the range $1400-1000 \mathrm{~cm}^{-1}$, were overlapped by the bands of polymers.

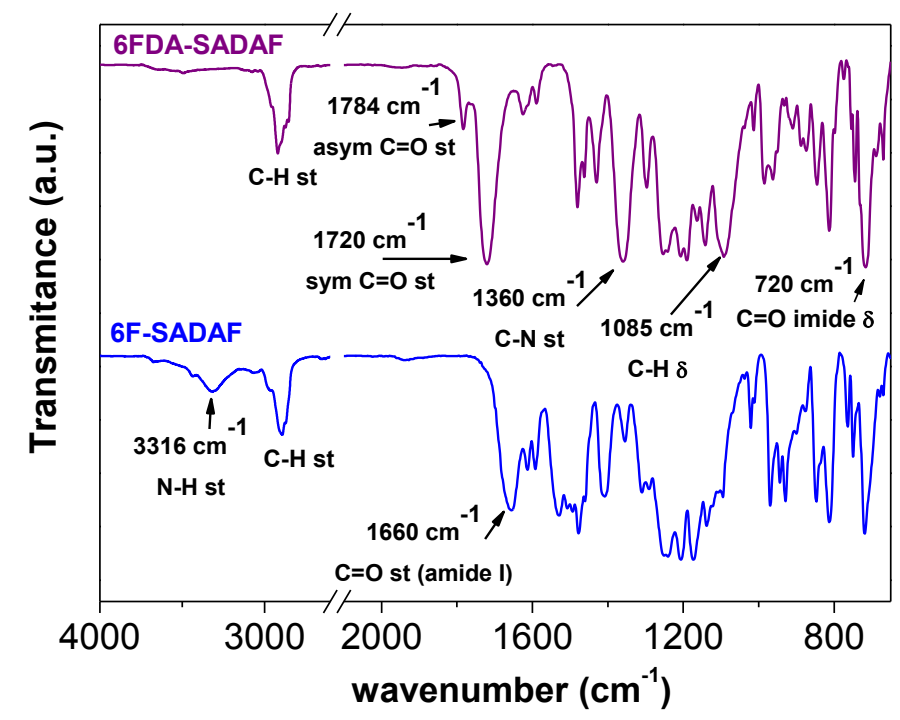

Fig. 2. ATR-FTIR of 6FDA-SADAF (polyimide film, thickness of $48 \mu$ ) and 6FSADAF films (polyamide film; thickness of $51 \mu$ )

\subsection{Physical and thermal properties of PIs and PAs}


Some important thermal and physical properties of polyimides and polyamides are listed in Table 1.

The weight-average molecular weights $\left(\mathrm{M}_{\mathrm{w}}\right)$, number-average molecular weights $\left(\mathrm{M}_{\mathrm{n}}\right)$, polydispersity indexes $\left(\mathrm{PI}=\mathrm{M}_{\mathrm{w}} / \mathrm{M}_{\mathrm{n}}\right)$ and intrinsic viscosity [ $\eta$ ] of soluble polymers, polyimide 6FDA-SADAF and polyamides 6F-SADAF and IP-SADAF were calculated from SEC measurements. The other two polyimides, derived from BPDA and PMDA, formed through thermal imidization were completely insoluble in any organic solvent. The molecular weight of polyimide was considerably higher than those of polyamides. In all cases, the polydispersity was high, indicating that there is a high content of low molecular weight components. The brittleness of films obtained by casting of polyamide IP-SADAF was consistent with the low molecular weight, which was probably a consequence of the rigidity of both monomers. For instance, the synthesis of the rod-like polyamide from SADAF and terephthaloyl chloride was tried using several methodologies. However, a very premature precipitation of the polymer was always attained.

All polymers showed excellent thermal stability in nitrogen atmosphere, with onset degradation temperatures above $450{ }^{\circ} \mathrm{C}$, and high char yields as it corresponds to highly aromatic polymers. Polyimides derived from PMDA and BPDA showed the highest thermal stability, close to $500{ }^{\circ} \mathrm{C}$. Differential TGA curves of PIs exhibited two or three degradation steps, as seen in Fig. 3. The first step in all PIs occurred from around $450{ }^{\circ} \mathrm{C}$ to about $550{ }^{\circ} \mathrm{C}$, and could be associated with the loss of the adamantane moiety that should vary from $20 \%$ to $29 \%$ depending on the dianhydride. An accurate determination of the first loss amount could not be made due to the overlap of the second loss. Yet, the experimental weight losses of the first step were around 5\% lower than the theoretical weight of the adamantane unit. For polyimides from BPDA and PMDA, the second weight loss occurred from $550{ }^{\circ} \mathrm{C}$ to $700{ }^{\circ} \mathrm{C}$ and it was associated with the generalized polymer degradation. For polyimide 6FDA-SADAF, an additional weight loss was observed from around $530{ }^{\circ} \mathrm{C}$ to about $600{ }^{\circ} \mathrm{C}$, which could be related to the loss of $\mathrm{CF}_{3}$ groups, followed by the complete polymer degradation. Therefore, it can be stated that the degradation mechanism depended on the dianhydride but, in all cases, the process started with the degradation of adamantane moiety.

Polyamides also showed excellent thermal stability and high char yields at 800 ${ }^{\circ} \mathrm{C}\left(\mathrm{R}_{800}\right)$ as expected, but somewhat lower than that of PIs. The degradation mechanism occurred in one step for IP-SADAF or in two steps for 6F-SADAF. The small 
differences observed in $T_{d}$ could be attributed to the fact that the decomposition started with the thermal breakdown of adamantane moiety as well. Yet, the extensive degradation of backbone happened virtually at the same time. The first weight loss of 6 F-SADAF was about twice the value that would be expected from the degradation of adamantane moiety (22\%).
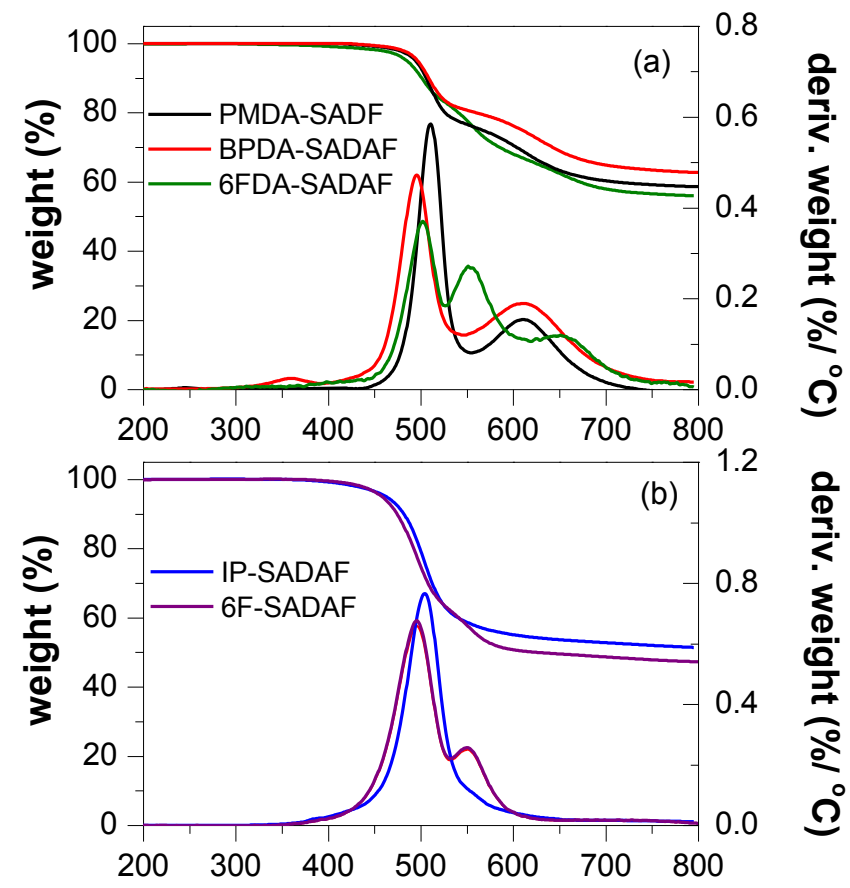

Temperature $\left({ }^{\circ} \mathrm{C}\right)$

Fig. 3. TGA ( $\mathrm{N}_{2}$ atmosphere) of SADAF polyimide films (up) and SADAF polyamide films (bottom)

Glass transition temperatures, $\mathrm{T}_{\mathrm{g}}$, for these polymers were determined by DSC. The samples were subjected to a heating-cooling-heating cycle in order to remove traces of solvent in the first heating. As can be seen from Table 1, all polymers exhibited high $\mathrm{T}_{\mathrm{g}}$, above $350{ }^{\circ} \mathrm{C}$. $\mathrm{T}_{\mathrm{g}} \mathrm{s}$ of polyimides derived from PMDA and BPDA were not detected by DSC measurements up to $400{ }^{\circ} \mathrm{C}$, probably due to the thermal imidization method that could cause crosslinking, and to the high rigidity of these structures. The size of the 
change in the specific heat at the glass transition, $\triangle C p$, was higher for PAs, which could suggest a lower rigidity of the backbone for these polymers.

Table 1 Physical and thermal properties of polyimides and polyamides

\begin{tabular}{cccccccc}
\hline Acronym & $\begin{array}{c}M w \\
(\text { Daltons) }\end{array}$ & PDI & $\begin{array}{c}{[\eta]} \\
(\mathbf{d L} / \mathbf{g})\end{array}$ & $\begin{array}{c}\boldsymbol{T}_{\boldsymbol{d}} \\
\left({ }^{\mathbf{0}} \mathbf{C}\right)\end{array}$ & $\begin{array}{c}\mathbf{R}_{800} \\
(\%)\end{array}$ & $\begin{array}{c}\boldsymbol{T}_{\boldsymbol{g}} \\
\left({ }^{\mathbf{0}} \mathbf{C}\right)\end{array}$ & $\begin{array}{c}\Delta C \boldsymbol{C} \\
(\mathbf{J} / \mathbf{g K})\end{array}$ \\
\hline PMDA-SADAF & insoluble & - & - & 490 & 58 & n.d. & \\
BPDA-SADAF & insoluble & - & - & 490 & 63 & n.d. & \\
6FDA-SADAF & 216,000 & 3.8 & 0.86 & 480 & 56 & 370 & 0.14 \\
6F-SADAF & 91,200 & 3.1 & 0.77 & 460 & 47 & 360 & 0.32 \\
IP-SADAF & 65,000 & 4.4 & 0.46 & 475 & 51 & 350 & 0.25 \\
\hline
\end{tabular}

*n.d., not detected by DSC

The chain packing of polymer films was evaluated from X-ray diffraction measurements. The Fig. 4 compares the WAXS patterns of polyimides (a) and polyamides (b), as a function of $2 \Theta$, at room temperature. The patterns did not show well-defined narrow reflections, which reflected the amorphous nature of these polymers. It is known that the most probable intersegmental distance between chains is typically related to the maximum of amorphous halo, as calculated from Bragg's Equation $(\lambda=2 \mathrm{~d} \sin \Theta$, being $\mathrm{d}$ the $\mathrm{d}$-spacing, $\Theta$ the scattering angle and $\lambda$ the wavelength). Regarding polyimides, the maximum position and the shape of amorphous halo were dependent on dianhydride monomer. The maxima at $18.0^{\circ}, 17.5^{\circ}$ and $16.2^{\circ}$ for polyimides derived from PMDA, BPDA and 6FDA corresponded to intersegmental distances of $0.49 \mathrm{~nm}, 0.51 \mathrm{~nm}$ and $0.55 \mathrm{~nm}$, respectively. Therefore, 6FDA-SADAF showed a higher contribution of larger intersegmental distances to the global scattering pattern. As to polyamides, the behavior was similar to that observed in polyimides with d-spacings of $0.56 \mathrm{~nm}$ and $0.49 \mathrm{~nm}$ for $6 \mathrm{~F}-\mathrm{SADAF}$ and IP-SADAF, the bulky $6 \mathrm{~F}$ moiety leading to the polyamide with the lower chain packing. Therefore, these results suggest that polyimide 6FDA-SADAF and polyamide 6F-SADAF should give membranes with the highest FFV. In Table 1 of the supporting information section, the values of diffraction angles and maximum of the amorphous halo are depicted. 

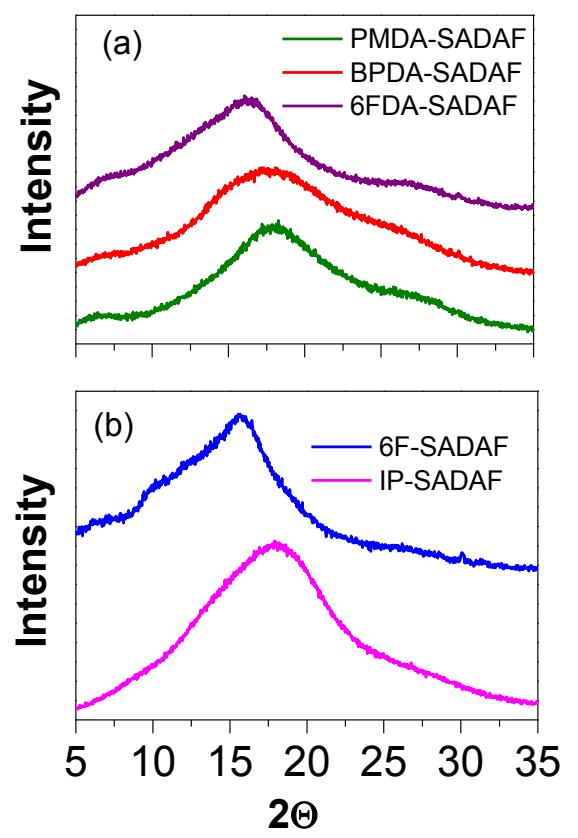

Fig. 4. WAXS diffractogram of SADAF polyimide films (up) and SADAF polyamide films (bottom)

Mechanical properties of 6F-SADAF polyamide and 6FDA-SADAF polyimide were excellent with tensile strength higher than $80 \mathrm{MPa}$ and mechanical moduli above 1.8 GPa. Also the elongation yield was high enough (higher than 10\%) to consider though to these polymers. However, the polyimides from PMDA and BPDA were brittle (elongations below 3\%), probably due to the high rigidity of these polyimides and also to the large conformational change produced by the thermal conversion from the polyamic acid to the corresponding polyimide. Regarding the IP-SADAF polyamide, the relatively medium-low molecular weight was translated to a cloudy film having mechanical properties much lower than that of the 6F-SADAF polymer (elongation at break lower than 5\% and tensile strength below $50 \mathrm{MPa}$ ). When this polymer was tested as gas separation membrane, the polymer film got broken inside the cell during the measurement. 


\subsection{Evaluation of 6FDA-SADAF and 6F-SADAF as gas separation membranes}

Due to the better gas separation properties frequently observed for polymers having hexafluoroisopropylidene groups, only the polymers derived from 6FDA and 6FC were evaluated. Furthermore, 6F-SADAF and 6FDA-SADAF showed good mechanical properties and high solubility in many organic solvents and because of that they could be easily processed in form of tough films by solution casting. The gas permeabilities and selectivities for both polymers are listed in Table 2. Permeability values for the polyimide, for all the five gases tested, were about two-fold higher than those of the polyamide, in agreement with its higher FFV. However, in spite of a higher diffusivity value, the main contribution to the increase in permeability seems to come from an increase in solubility. The significant augment in permeability of PI was accompanied by an improvement in selectivity, especially for the gas pair $\mathrm{CO}_{2} / \mathrm{CH}_{4}$.

Table 2.- Permeability values, $P\left(\right.$ Barrer $\left.^{\mathrm{a}}\right)$, Diffusion coefficients, $D\left(10^{-8} \mathrm{~cm}^{2} \cdot \mathrm{s}^{-1}\right)$, Solubility coefficients, $\mathrm{S}\left(10^{-2} \mathrm{~cm}^{3}(\mathrm{STP}) \cdot \mathrm{cm}^{-3} \cdot \mathrm{cmHg}^{-1}\right)$, and Selectivity coefficients $(\alpha)$ of polyimide 6FDA-SADAF and polyamide 6F-SADAF membranes.

\begin{tabular}{ccccccccccc}
\hline Membrane & Parameter & $\mathrm{He}$ & $\mathrm{O}_{2}$ & $\mathrm{~N}_{2}$ & $\mathrm{CH}_{4}$ & $\mathrm{CO}_{2}$ & $\alpha \mathrm{O}_{2} / \mathrm{N}_{2}$ & $\alpha \mathrm{CO}_{2} / \mathrm{N}_{2}$ & $\alpha \mathrm{CO}_{2} / \mathrm{CH}_{4}$ \\
\hline \multirow{2}{*}{ 6FDA-SADAF } & $\mathrm{P}$ & 87 & 7.3 & 1.21 & 0.61 & 31 & 6.2 & 26 & 51 \\
& $\mathrm{D}$ & -- & 3.3 & 0.75 & 0.12 & 1.35 & 4.4 & 1.8 & 11 \\
& $\mathrm{~S}$ & -- & 2.2 & 1.6 & 5.1 & 23.0 & 1.3 & 14 & 4.8 \\
6F-SADAF & $\mathrm{P}$ & 41 & 3.4 & 0.60 & 0.34 & 14 & 5.7 & 23 & 41 \\
& $\mathrm{D}$ & -- & 2.5 & 0.58 & 0.10 & 0.95 & 4.3 & 1.6 & 9.5 \\
& $\mathrm{~S}$ & -- & 1.4 & 1.0 & 3.4 & 14.7 & 1.4 & 15 & 4.3
\end{tabular}

a) Barrer $=10^{-10}\left(\mathrm{~cm}^{3}(\mathrm{STP}) \cdot \mathrm{cm}\right)\left(\mathrm{cm}^{-2} \cdot \mathrm{s}^{-1} \cdot \mathrm{cmHg}^{-1}\right)=7.5005 \cdot 10^{-18} \mathrm{~m}^{2} \mathrm{~s}^{2} \mathrm{~Pa}^{-1}$ (SI units)

Density 6FDA-SADAF: $1.3407 \pm 0.0001 \mathrm{~g} / \mathrm{cm}^{3} ; \mathrm{FFV}=0.180$.

Density 6F-SADAF: $1.3201 \pm 0.0001 \mathrm{~g} / \mathrm{cm}^{3} ; \mathrm{FFV}=0.157$.

The productivity of gas separation for $\mathrm{CO}_{2} / \mathrm{CH}_{4}$, which is represented in Fig. 5 along with the 1991 and 2008 Robeson limits, was superior to other polyimides bearing adamantyl ester pendent groups[6] or 9,9'-fluorene moieties[40,41] and also to some polyamides containing bulky side groups[9,42]. Specially, the polyimide 6FDA- 
SADAF was placed very close to the Robeson upper bound of 1991[43]. Overpassing the 1991 for common glassy polymers is infrequent, and only well-designed materials as PIMs, TR materials, some polypyrrolones and other specific aromatic polymers are able to approach to the 2008 Robeson limit[44-47]. Nevertheless, the values shown in this work determine these materials to have excellent and balanced properties for gas separation applications. The synthesis of other materials having the spiro-(adamantanefluorene) is being designed for the field of gas separation membranes and future work will be published elsewhere.

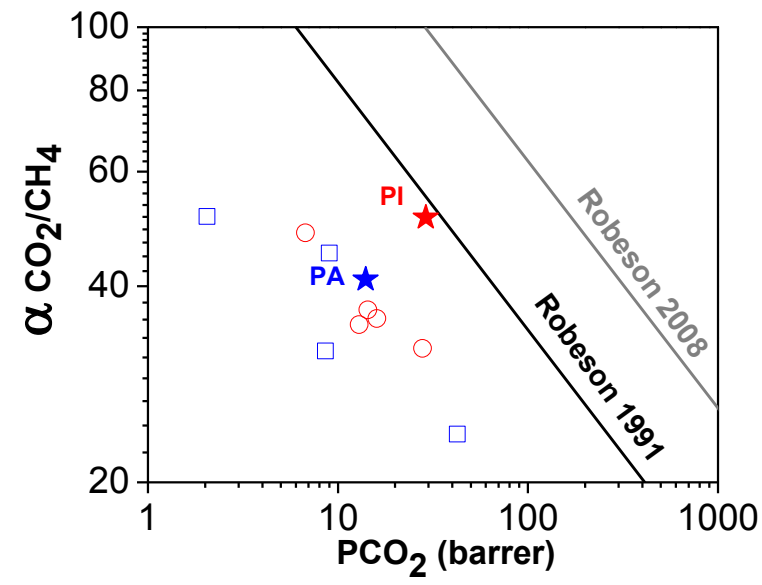

Fig. 5- Gas performance of 6FDA-SADAF (red star) and 6F-SADAF (blue star) for $\mathrm{CO}_{2} / \mathrm{CH}_{4}$ separation compared to other PIs (red circles) and PAs (blue squares) reported in the literature[6,34,35,45,48].

\section{Conclusions}

A new aromatic diamine monomer has been designed and obtained using a straightforward methodology. This monomer bears a bulky adamantane group and a spiro carbon, which connects the 2-positions of an adamantane moiety to a 9,9'-fluorene group. This combination provides a monomer having a high rigidity and a very bulky group. The synthesis of SADAF could be accomplished by using a flexible methodology. The combination of this monomer with two acid chlorides, and three commercial dianhydrides has produced two sets of aromatic polyamides and polyimides with high molecular weight. 
Regarding the polyimides, only the polymer coming from 6FDA was soluble both as polyamic acid and as polyimide. The other two ones, arising from PMDA and BPDA, came out during the chemical imidization process, what forced us to employ the thermal imidization methodology.

TGA denoted that these materials have excellent thermal resistance with degradation temperature values higher than $450{ }^{\circ} \mathrm{C}$. With regards to $\mathrm{T}_{\mathrm{g}}$, the values were higher than $350{ }^{\circ} \mathrm{C}$ for aromatic polyamides and higher than $370{ }^{\circ} \mathrm{C}$ for the polyimide from 6FDA. It was not possible to appreciate the presence of any $T_{g}$ for the thermally imidized polyimides.

Mechanical properties showed values similar to common aromatic polyamides and polyimides, which permit to use these polymers for applications where high pressures are required; for instance in gas separation applications. Polymers, as denoted by WAXS, showed an amorphous behavior, and the presence of the hexafluoroisopropylidene group shifted the maximum of the amorphous halo to higher values of d-spacing.

Due to the better gas separation values observed for polyamides and polyimides having the hexafluoroisopropylidene moieties, and due also to their higher d-spacing value, 6FDA-SADAF and 6F-SADAF were measured as membranes. The values of permeability and selectivity were very interesting because the polymeric membranes showed well-balanced gas productivity, in particular for the $\mathrm{CO}_{2} / \mathrm{CH}_{4}$ separation.

\section{Acknowledgments}

The financial support provided, by the MINECO (MAT2011-25513, MAT201020668, and MAT2013-45071-R) is gratefully acknowledged. This research was also supported by the Korea Carbon Capture \& Sequestration R\&D Center (KCRC) through the National Research Foundation of Korea (NRF) funded by the Ministry of Science, ICT, and Future Planning (NRF-2014M1A8A1049305). We are also indebted to the Spanish Junta de Castilla y Leon for its funding help (project VA248U13). Authors kindly acknowledge Sara Rodriguez by carrying out several gas separation tests.

\section{Supporting Information}

In this section, the detailed synthesis of SADAF, NMR $\left({ }^{1} \mathrm{H}\right.$ and $\left.{ }^{13} \mathrm{C}\right)$ spectra of SADAF, ${ }^{1} \mathrm{H}-\mathrm{NMR}$ spectrum of 6FDA-SADAF and a Table with the WAXS characteristics of SADAF polymers have been included. 
[1] Sroog CE. Polyimides. Prog Polym Sci 1991;16:561-694.

[2] Wanek E. Polyimides, synthesis, characterization, and applications, Volume 1. K. L. Mittal, Ed., Plenum, New York, 1984, 614 pp. Price: \$89.50. J Polym Sci Part C Polym Lett 1986;24:295-6.

[3] Liaw DJ, Wang KL, Huang YC, Lee KR, Lai JY, Ha CS. Advanced polyimide materials: Syntheses, physical properties and applications. Prog Polym Sci 2012;37:907-74.

[4] Abajo J De, Campa JG De. Processable Aromatic Polyimides 1999;140.

[5] De Abajo J, De La Campa JG, Lozano AE, Alvarez JC. Thermally stable polymers: Novel aromatic polyamides. Adv Mater 1995;7:148-51.

[6] Maya EM, Garcia-Yoldi I, Lozano AE, de La Campa JG, de Abajo J. Synthesis, characterization, and gas separation properties of novel copolyimides containing adamantyl ester pendant groups. Macromolecules 2011;44:2780-90.

[7] Wiegand JR, Smith ZP, Liu Q, Patterson CT, Freeman BD, Guo R. Synthesis and characterization of triptycene-based polyimides with tunable high fractional free volume for gas separation membranes. J Mater Chem A 2014;2:13309.

[8] Morisato A, Ghosal K, Freeman BD, Chern RT, Alvarez JC, de la Campa JG, et al. Gas separation properties of aromatic polyamides containing hexafluoroisopropylidene groups. J Memb Sci 1995;104:231-41.

[9] Singh A, Ghosal K, Freeman BD, Lozano AE, de la Campa JG, de Abajo J. Gas separation properties of pendent phenyl substituted aromatic polyamides containing sulfone and hexafluoroisopropylidene groups. Polymer (Guildf) 1999;40:5715-22.

[10] Calle M, Lozano AE, de La Campa JG, de Abajo J. Novel Aromatic Polyimides Derived from 5'- t -Butyl-2'-pivaloylimino-3,4,3",4"'- $\mathrm{m}$-terphenyltetracarboxylic Dianhydride with Potential Application on Gas Separation Processes. Macromolecules 2010;43:2268-75.

[11] Calle M, Lozano AE, de Abajo J, de la Campa JG, Álvarez C. Design of gas separation membranes derived of rigid aromatic polyimides. 1. Polymers from diamines containing di-tert-butyl side groups. J Memb Sci 2010;365:145-53.

[12] Calle M, García C, Lozano AE, De la Campa JG, De Abajo J, Álvarez C. Local chain mobility dependence on molecular structure in polyimides with bulky side groups: Correlation with gas separation properties. J Memb Sci 2013;434:121-9.

[13] Santiago-García JL, Álvarez C, Sánchez F, de la Campa JG. Gas transport properties of new aromatic polyimides based on 3,8-diphenylpyrene-1,2,6,7tetracarboxylic dianhydride. J Memb Sci 2015;476:442-8. 
[14] Kim K-J, So W-W, Moon S-J. Preparation of 6FDA-based polyimide membranes for CO2 gas separation. Stud Surf Sci Catal 2004;153:531-4.

[15] Robeson LM, Smith ZP, Freeman BD, Paul DR. Contributions of diffusion and solubility selectivity to the upper bound analysis for glassy gas separation membranes. J Memb Sci 2014;453:71-83.

[16] Freeman BD. Basis of Permeability/Selectivity Tradeoff Relations in Polymeric Gas Separation Membranes. Macromolecules 1999;32:375-80.

[17] Fort RC, Schleyer P von R. Adamantane: Consequences of the Diamondoid Structure. Chem Rev 1964;64:277-300.

[18] Ferrero E, Espeso JF, de la Campa JG, de Abajo J, Lozano AE. Synthesis and characterization of aromatic polyamides containing alkylphthalimido pendent groups. J Polym Sci Part A Polym Chem 2002;40:3711-24.

[19] Khardin AP, Radchenko SS. Polymeric Derivatives of Adamantane. Russ Chem Rev 1982;51:272.

[20] Espeso JF, Lozano ÁE, de La Campa JG, García-Yoldi Í, de Abajo J. Synthesis and properties of new aromatic polyisophthalamides with adamantylamide pendent groups. J Polym Sci Part A Polym Chem 2010;48:1743-51.

[21] Bera D, Bandyopadhyay P, Ghosh S, Banerjee S. Gas transport properties of aromatic polyamides containing adamantyl moiety. J Memb Sci 2014;453:17591.

[22] Mathews AS, Kim I, Ha CS. Fully aliphatic polyimides from adamantane-based diamines for enhanced thermal stability, solubility, transparency, and low dielectric constant. J Appl Polym Sci 2006;102:3316-26.

[23] Guiver MD, Lee YM. Polymer Rigidity Improves Microporous Membranes. Science (80- ) 2013;339:284-5.

[24] Li S, Jo HJ, Han SH, Park CH, Kim S, Budd PM, et al. Mechanically robust thermally rearranged (TR) polymer membranes with spirobisindane for gas separation. J Memb Sci 2013;434:137-47.

[25] Bezzu CG, Carta M, Tonkins A, Jansen JC, Bernardo P, Bazzarelli F, et al. A spirobifluorene-based polymer of intrinsic microporosity with improved performance for gas separation. Adv Mater 2012;24:5930-3.

[26] Dewar MJS, Zoebisch EG, Healy EF, Stewart JJP. Development and use of quantum mechanical molecular models. 76. AM1: a new general purpose quantum mechanical molecular model. J Am Chem Soc 1985;107:3902-9.

[27] Lozano AE, De La Campa JG, De Abajo J. Aromatic polyamides and polyimides derived from 3,3?-diaminobiphenyl: Synthesis, characterization, and molecular simulation study. J Polym Sci Part A Polym Chem 1999;37:4646-55. 
[28] Kim YH, Kim HS, Kwon SK. Synthesis and characterization of highly soluble and oxygen permeable new polyimides based on twisted biphenyl dianhydride and spirobifluorene diamine. Macromolecules 2005;38:7950-6.

[29] Wen P., Kim Y., Chun H., Yang S-Y., Lee M-H. c. Syntheses and characterizations of cardo polyimides based on new spirobifluorene diamine monomer. Mater Chem Phys 2013;139:923-30.

[30] Lozano AE, de Abajo J, de la Campa JG. Synthesis of aromatic polyisophthalamides by in situ silylation of aromatic diamines. Macromolecules 1997;30:2507-8.

[31] Lozano AE, de Abajo J, la Campa JG. Quantum semiempirical studies on the formation of aromatic polyamides, 1 . Effect of structural parameters of diamines on the polyamidation reaction. Macromol Theory Simulations 1993;2:815-27.

[32] Muñoz DM, de la Campa JG, de Abajo J, Lozano AE. Experimental and Theoretical Study of an Improved Activated Polycondensation Method for Aromatic Polyimides. Macromolecules 2007;40:8225-32.

[33] Muñoz DM, Calle M, Lozano AE, Campa JG De. An improved method of preparing high molecular weight polyimides. base-assisted in situ silylation of diamines 2008;49:723-4.

[34] Recio R, Palacio L, Pradanos P, Hernandez a, Lozano a, Marcos a, et al. Gas separation of 6FDA-6FpDA membranesEffect of the solvent on polymer surfaces and permselectivity. J Memb Sci 2007;293:22-8.

[35] Bos A, Pünt IGM, Wessling M, Strathmann H. Plasticization-resistant glassy polyimide membranes for CO2/CO4 separations. Sep Purif Technol 1998;14:2739.

[36] Bandyopadhyay P, Bera D, Banerjee S. Synthesis, characterization and gas transport properties of semifluorinated new aromatic polyamides. Sep Purif Technol 2013;104:138-49.

[37] Smith ZP, Czenkusch K, Wi S, Gleason KL, Hernández G, Doherty CM, et al. Investigation of the chemical and morphological structure of thermally rearranged polymers. Polymer (Guildf) 2014;55:6649-57.

[38] HyperChem(TM), Professional 8.0.3 Hypercube, Inc., Version 8.0.3, Florida, USA n.d.

[39] Munoz DM, Lozano a. E, De La Campa JG, De Abajo J. Monomer Reactivity and Steric Factors affecting the Synthesis of Aromatic Polyamides. High Perform Polym 2007;19:592-602.

[40] Kazama S., Teramoto T., Haraya K. Oxygen separation properties of bis(phenyl)fluorene-based cardo polymer membranes. High Perform Polym 2005;17:3-17. 
[41] Kazama S, Teramoto T, Haraya K. Carbon dioxide and nitrogen transport properties of bis(phenyl)fluorene-based cardo polymer membranes. J Memb Sci 2002;207:91-104.

[42] Sato S, Ose T, Miyata S, Kanehashi S, Ito H, Matsumoto S, et al. Relationship between the gas transport properties and the refractive index in high-free-volume fluorine-containing polyimide membranes. J Appl Polym Sci 2011;121:2794803.

[43] Robeson LM. Correlation of separation factor versus permeability for polymeric membranes. J Memb Sci 1991;62:165-85.

[44] Robeson LM, Freeman BD, Paul DR, Rowe BW. An empirical correlation of gas permeability and permselectivity in polymers and its theoretical basis. J Memb Sci 2009;341:178-85.

[45] Rogan Y, Malpass-Evans R, Carta M, Lee M, Jansen JC, Bernardo P, et al. A highly permeable polyimide with enhanced selectivity for membrane gas separations. Chem Eng Res Bull 2014;2:4874.

[46] Rose I, Carta M, Malpass-Evans R, Ferrari M-C, Bernardo P, Clarizia G, et al. Highly Permeable Benzotriptycene-Based Polymer of Intrinsic Microporosity. ACS Macro Lett 2015;0:912-5.

[47] Carta M, Croad M, Jansen JC, Bernardo P, Clarizia G, McKeown NB. Synthesis of cardo-polymers using Tröger's base formation. Polym Chem 2014;5:5255.

[48] Carta M, Malpass-Evans R, Croad M, Rogan Y, Jansen JC, Bernardo P, et al. An efficient polymer molecular sieve for membrane gas separations. Science 2013;339:303-7. 
